\title{
Fractura del cuerpo del astrágalo en un niño: a propósito de un caso
}

\author{
Tamara Rodríguez-López, Alberto Núñez Medina \\ Unidad de Pie y Tobillo, Servicio de Traumatología, Hospital Universitario de Torrejón de Ardoz, Madrid, España
}

\begin{abstract}
RESUMEN
La fractura del cuerpo del astrágalo es un cuadro extremadamente raro en la población infantil, y se asocia con mecanismos de alta energía. Se trata de una lesión muy grave y tiene un manejo complejo para el cirujano ortopedista, no exento de complicaciones, como la necrosis avascular, la artrosis postraumática o la seudoartrosis. Presentamos a un niño de 10 años con una fractura-luxación del cuerpo del astrágalo, su manejo de urgencia, el tratamiento definitivo y la evaluación clínico-radiológica al año de la lesión.
\end{abstract}

Palabras clave: Fractura-luxación; astrágalo; necrosis.

Nivel de Evidencia: IV

Talar body fracture in a 10-year-old boy. Case presentation

\section{ABSTRACT}

Talar body fractures are an extremely rare presentation in children that are associated with high-energy trauma. They constitute devastating injuries and a management challenge for orthopedic surgeons, which could be further complicated by avascular necrosis, post-traumatic arthritis, and non-union. We report a case of a fracture-dislocation of the talar body in a 10-year-old boy, including emergency management, definitive treatment, and 1-year follow-up clinical and radiologic findings.

Key words: Fracture-dislocation; talus, necrosis.

Level of Evidence: IV

\section{INTRODUCCIÓN}

Las fracturas de astrágalo son extremadamente raras en la infancia, debido a la inmadurez ósea y a la mayor laxitud del pie infantil. ${ }^{1}$ La creciente participación de los niños en actividades de impacto determina que la prevalencia de este tipo de fracturas vaya en aumento. En algunos casos, como en la escalada, el tobillo se queda fijo en posición neutra, mientras que el impacto del calcáneo anula la hiperflexión dorsal en el tobillo. De esta manera, si la fuerza agresora continúa, se produce una lesión en el cuerpo del astrágalo que puede o no llevar a la luxación de las articulaciones próximas. ${ }^{2}$

La clasificación más empleada es la de Marti que considera el grado de desplazamiento y luxación inicial, así como el número de articulaciones afectadas. Si hay una luxación articular acompañante, la tasa de necrosis avascular y artrosis postraumática aumenta, de forma notable, en el adulto (Tabla). ${ }^{3}$

Tabla. Clasificación de Marti para fracturas

\begin{tabular}{|l|l|}
\hline Tipo I & Fractura del cuello/la cabeza del astrágalo \\
\hline Tipo II & Fractura de la cabeza o el cuerpo del astrágalo, sin desplazamiento \\
\hline Tipo III & Luxación de la cabeza o el cuerpo del astrágalo con luxación articular acompañante \\
\hline Tipo IV & Fractura del cuello o el cuerpo del astrágalo con luxación completa del cuerpo o fractura conminuta \\
\hline
\end{tabular}
Recibido el 15-10-2019. Aceptado luego de la evaluación el 1-3-2020 • Dra. TAMARA RODRÍGUEZ-LÓPEZ • tamara.rl85@ gmail.com ID https://orcid.org/0000-0003-0650-9265
Cómo citar este artículo: Rodríguez-López T, Núñez Medina A. Fractura del cuerpo del astrágalo en un niño: a propósito de un caso. Rev Asoc Argent Ortop Traumatol 2020;85(4):387-392.
https://doi.org/10.15417/issn. 1852-7434.2020.85.4.1024 
Presentamos un caso clínico de una fractura-luxación de astrágalo en un niño provocada por un mecanismo de alta energía con compresión neurovascular preoperatoria. El tratamiento quirúrgico de urgencia permite aliviar la compresión y evitar complicaciones articulares. ${ }^{4}$

\section{CASO CLÍNICO}

Niño de 10 años sin antecedentes de interés, que sufrió un traumatismo no especificado en el tobillo izquierdo mientras hacía escalada. Acudió al departamento de urgencias con el tobillo fijo en inversión y flexión neutra. En el examen físico, se detectó un déficit sensitivo del nervio tibial posterior en aumento, con pulsos; no se detectaron lesiones de partes blandas asociadas.

En los estudios por imágenes iniciales, se observó una fractura-luxación del cuerpo del astrágalo. Luego de la tomografía computarizada, se clasificó a la fractura como de tipo III de la clasificación de Marti (Figura 1).
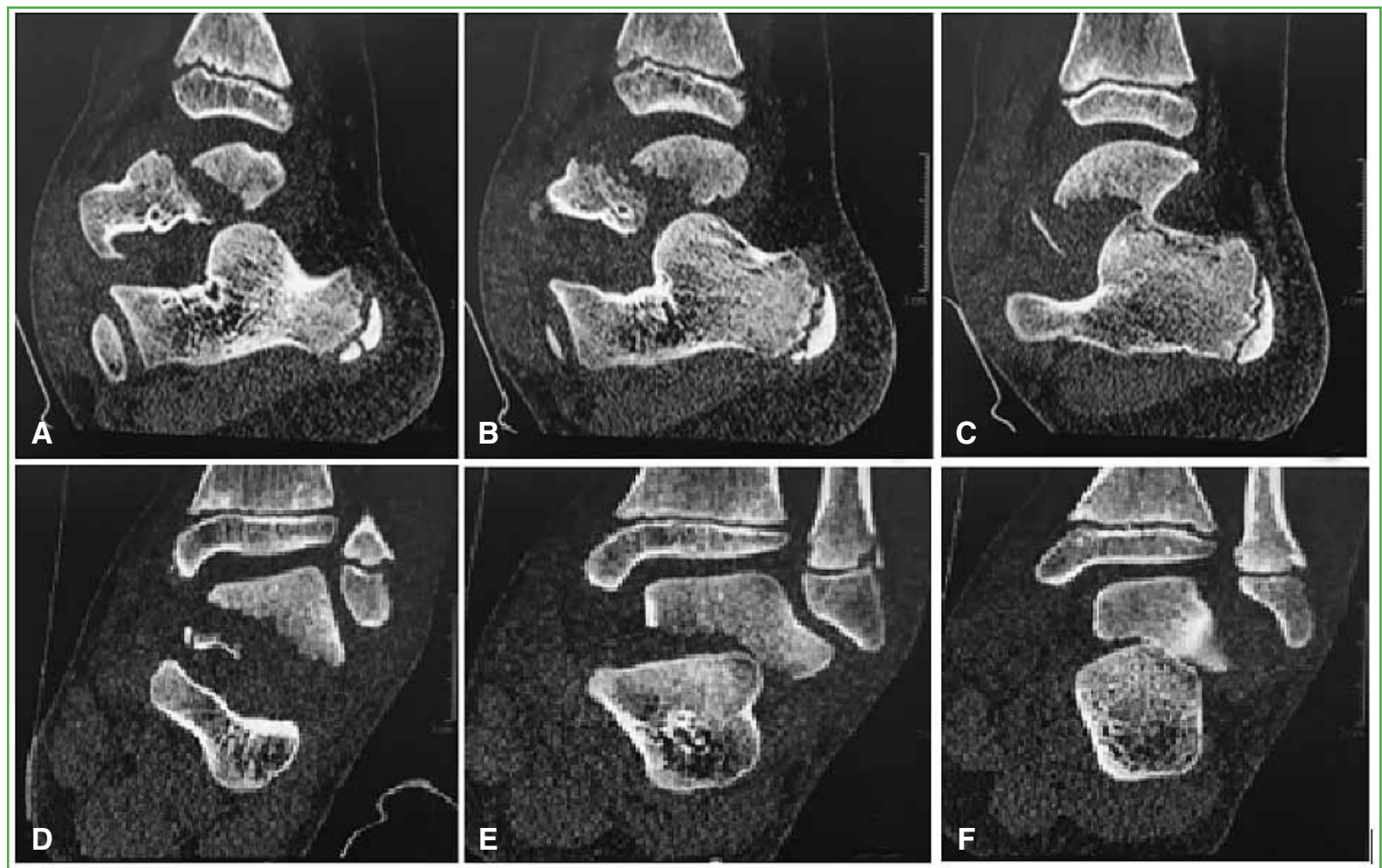

Figura 1. Tomografía computarizada preoperatoria. A-C. Corte sagital. D-F. Corte coronal.

Ante el empeoramiento sensitivo progresivo y el tipo de lesión, se decidió el tratamiento quirúrgico de urgencia. Tras la anestesia general y la administración de antibióticos profilácticos, se procedió a la reducción abierta de la luxación subastragalina y a la revisión del paquete vasculonervioso por un abordaje medial. Durante la exploración, se observó la indemnidad del paquete vasculonervioso, aunque presentaba un importante hematoma en el túnel tarsiano que se eliminó para evitar una mayor compresión. Tras reducir la articulación subastragalina por vía medial, se redujo, de forma abierta, la fractura por doble vía (medial y lateral) sintetizándola temporalmente con dos agujas de Kirschner. Una vez comprobada la reducción mediante un control radiográfico, se procedió a la síntesis definitiva con tornillos canulados a compresión de espira parcial de 4,0 (posterolateral y anteromedial) (Figura 2). 


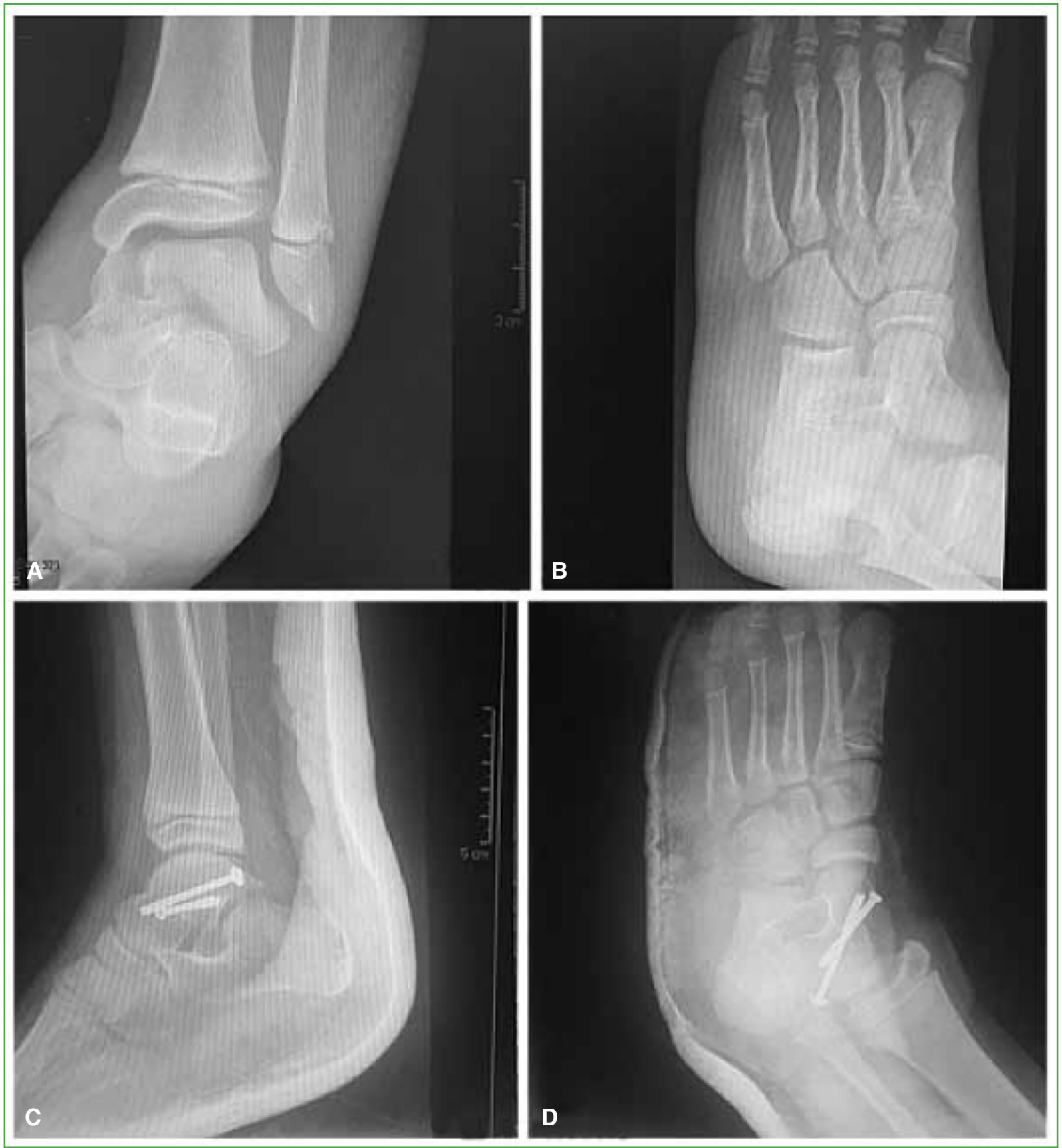

Figura 2. A y B. Radiografías preoperatorias de la fractura del cuerpo del astrágalo de tipo III de la clasificación de Marti. C y D. Control radiográfico posoperatorio.

\section{Seguimiento y resultados}

A las $48 \mathrm{~h}$ de la intervención, la sensibilidad de la planta del pie estaba completamente restablecida. Se mantuvo una inmovilización suropédica adicional durante tres semanas y, luego, se permitió la movilidad activa. Se autorizó la carga con la consolidación de la fractura (6 semanas).

Tras la rehabilitación, la movilidad del tobillo era completa y el paciente reanudó su vida normal a los tres meses, sin secuelas neurológicas evidentes. 
A los 10 meses de la lesión, se extrajo el material de osteosíntesis. Tras un año y medio de seguimiento, se repitieron las radiografías en carga y se realizó una valoración clínica empleando la escala de la American Orthopaedic Foot and Ankle Society (AOFAS) para pie y tobillo.

En el estudio radiográfico, se comprobó la consolidación completa de la fractura, así como la alineación normal del tobillo y el pie. No se detectaron cambios artrósicos ni necrosis avascular (Figura 3). El paciente no tenía síntomas y practicaba actividades deportivas de impacto sin complicaciones. El puntaje de la escala AOFAS fue de 95 al año y medio de la lesión (excelente 90-100, bueno 80-89, malo <60).
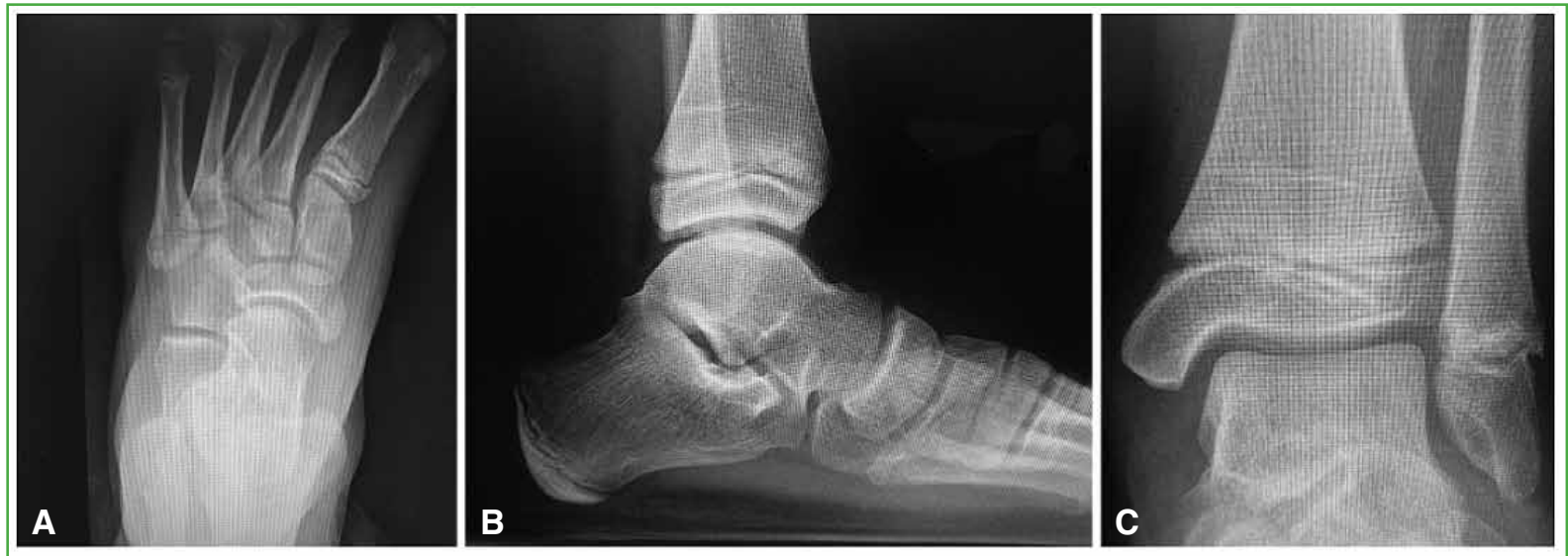

Figura 3. Control radiográfico al final del seguimiento, tras el retiro del material de osteosíntesis.

\section{DISCUSIÓN}

Las fracturas astragalinas suponen una lesión de extrema gravedad debido a que es el hueso conector entre el tobillo, el tarso y la articulación subastragalina. Diferenciamos entre fracturas del cuello o del cuerpo del astrágalo en función de la localización del trazo de fractura. Cuando el trazo llega hasta la articulación subastragalina se la considerará una fractura del cuerpo del astrágalo. 5

Las fracturas astragalinas son lesiones extremadamente raras en la población infantil debido a la elasticidad de los tejidos, la inmadurez ósea y la menor incidencia de lesiones por alta energía comparada con la de los adultos. No obstante, deportes de alta energía, como la escalada, o los accidentes de tránsito hacen que esta lesión no sea única de la población adulta. ${ }^{1}$

Se cree que el mecanismo de producción de una fractura del cuerpo astragalino es un impacto del calcáneo sobre un astrágalo fijo en posición neutra a causa de una caída de altura, como ocurre en la escalada. Si la fuerza agresora persiste se fractura el cuerpo astragalino y se luxan las articulaciones circundantes, principalmente la subastragalina. ${ }^{1,6-8}$ Este podría ser el mecanismo productor en nuestro paciente, pues el niño refirió una caída en la que intentó meter el pie en una saliente del rocódromo y quedó bloqueado en inversión y flexión neutra de tobillo sin poder realizar movimientos de ningún tipo tras el impacto.

Si bien las lesiones vasculonerviosas son raras, en el caso de grandes desplazamientos se pueden producir lesiones por compresión del paquete vasculonervioso. ${ }^{4,9}$ En nuestro paciente, aunque el desplazamiento no era demasiado llamativo, la sensibilidad disminuida de la planta del pie obligaba a realizar una exploración quirúrgica, en la que se observó un hematoma de gran tamaño que comprimía el nervio y que podría haber causado la hipoestesia.

Cuando hay alteraciones neurovasculares, grandes desplazamientos o lesiones tendinosas incarceradas, lo que parece claro es que no se deben efectuar maniobras intempestivas; se aconseja una revisión quirúrgica urgente de la lesión con síntesis definitiva si las partes blandas lo permiten.,

Dada la alta energía necesaria para producir este tipo de fracturas, no es extraño que haya lesiones de partes blandas y óseas asociadas. En estas situaciones, la fijación deberá hacerse mediante técnicas de fijación externa hasta que las partes blandas permitan una síntesis definitiva. En la bibliografía actual, no se informan diferencias en los resultados en cuanto a los tiempos de fijación, siempre y cuando se produzca una reducción precoz de la fractura para evitar la necrosis avascular. ${ }^{1,4,10,11}$ En nuestro paciente, aunque tenía un déficit sensitivo progresivo y el mecanismo de producción era de alta energía, las partes blandas estaban indemnes y esto facilitó el tratamiento definitivo. 
Kamphuis y cols. documentan lesiones fisarias asociadas a nivel del maléolo interno; en estos casos, esta lesión favorece el tratamiento quirúrgico del astrágalo, pues permite la reducción visualizada directamente desde dicho foco de fractura como si se tratase de una osteotomía. ${ }^{1,7}$

Es necesario efectuar una reducción perfectamente anatómica en este tipo de fracturas. Si la cúpula astragalina no se visualiza correctamente por vía lateral, se deberá optar por un abordaje anteromedial adicional evitando lesionar el aporte vascular de la vertiente medial del astrágalo., Aunque una osteotomía del maléolo interno aporta una visualización excepcional a este tipo de fracturas, decidimos no realizarla a fin de evitar complicaciones fisarias en la tibia distal, porque era un niño.

En nuestro caso, utilizamos un abordaje inicial anteromedial para reducir la articulación subastragalina y proceder a la liberación del paquete vasculonervioso. A continuación, realizamos un abordaje lateral adicional para conseguir una reducción anatómica de la fractura.

En la población pediátrica, el método de fijación más habitual son las agujas de Kirschner; no obstante, optamos por emplear tornillos de espira parcial para conseguir la compresión de la fractura. A los 10 meses de la lesión se programó su retiro para evitar complicaciones..$^{1,12}$

Las fracturas desplazadas del cuerpo astragalino se caracterizan por su alto riesgo de necrosis avascular en los adultos. En los niños, esto es menos común, dada la frecuencia más baja de luxación subastragalina asociada.

Eberl y cols. compararon el tipo de fracturas astragalinas en pacientes $<12$ años y $>12$ años, y hallaron que, para mecanismos de alta energía, la población $<12$ años tenía lesiones de menor complejidad (Marti I y II) que aquellos $>12$ años. ${ }^{13}$ Smith y cols. comunicaron que este tipo de lesiones se relacionaba más con adolescentes que con niños. Las complicaciones descritas son necrosis avascular (7\%), artrosis (17\%) y retraso de la consolidación (3\%). ${ }^{14} \mathrm{Tal}$ y como se refleja en los estudios antes comentados, la ausencia de luxación subastragalina y un metabolismo óseo aumentado (característico de la edad) favorecieron que no ocurriera ninguna de las complicaciones descritas. ${ }^{13-15}$

Nuestro paciente de 10 años sufrió una fractura del cuerpo del astrágalo, de tipo III de la clasificación de Marti. A pesar de su edad, la energía con la que se produjo el impacto fue más que suficiente para causar una lesión más grave que la habitual en este grupo etario. La mayor elasticidad de los tejidos y el metabolismo óseo aumentado propio de la edad fueron factores determinantes a la hora de evitar complicaciones.

\section{CONCLUSIONES}

Aunque las fracturas del cuerpo del astrágalo son lesiones poco frecuentes durante la infancia, la incidencia se está incrementando, porque la población infantil practica cada vez más actividades de impacto. Es un cuadro de extrema gravedad y de manejo complejo.

Nuestro paciente tenía una fractura-luxación del cuerpo del astrágalo de tipo III de Marti. El riesgo de complicaciones y secuelas por este tipo de fracturas es alto en la población adulta. El manejo urgente y la edad del paciente facilitaron el resultado satisfactorio del caso.

Conflicto de intereses: Los autores no declaran conflictos de intereses.

\section{BIBLIOGRAFÍA}

1. Kamphuis SJ, Meijs CM, Kleinveld S, Diekerhof CH, van der Heijden FH. Talar fractures in children: a possible injury after go-karting accidents. J Foot Ankle Surg 2015;54(6):1206-12. https://doi.org/10.1053/j.jfas.2015.07.013

2. Sneppen O, Buhl O. Fracture of the talus. A study of its genesis and morphology based upon cases with associated ankle fracture. Acta Orthop Scand 1974;45(2):307-20. https://doi.org/10.3109/17453677408989151

3. Rammelt S, Zwipp H. Talar neck and body fractures. Injury 2009;40(2):120-35. https://doi.org/10.1016/j.injury.2008.01.021 
4. Kizilay YO, Aytan O. Low-energy Hawkins type III talar neck fracture-dislocation with neurovascular and tendon entrapment in a pediatric patient. J Foot Ankle Surg 2017;56(6):1288-91. https://doi.org/10.1053/j.jfas.2017.05.007

5. Ebraheim NA, Patil V, Owens C, Kandimalla Y. Clinical outcome of fractures of the talar body. Int Orthop 2008;32(6):773-7. https://doi.org/10.1007/s00264-007-0399-5

6. Ely EE, Konstantakos EK, Laughlin RT, Johnson RM, Binski JC. Total dislocation of the talus and the navicular: a case report. J Orthop Trauma 2009;23(7):546-9. https://doi.org/10.1097/BOT.0b013e3181a18d7a

7. Buza JA 3rd, Leucht P. Fractures of the talus: current concepts and new developments. Foot Ankle Surg 2018;24(4):282-90. https://doi.org/10.1016/j.fas.2017.04.008

8. Jensen I, Wester JU, Rasmussen F, Lindequist S, Schantz K. Prognosis of fracture of the talus in children. 21 (7-34)year follow-up of 14 cases. Acta Orthop Scand 1994;65(4):398-400. https://doi.org/10.3109/17453679408995478

9. Barnett JR, Ahmad Ma, Khan W, O’Gorman A. The diagnosis, management and complications associated with fractures of the talus. Open Orthop J 2017;11:460-6. https://doi.org/10.2174/1874325001711010460

10. Vallier HA, Nork SE, Benirschke SK, Sangeorzan BJ. Surgical treatment of talar body fractures. J Bone Joint Surg Am 2003;85(9):1716-24. https://doi.org/10.2106/00004623-200309000-00010

11. Haverkort JJ, Leenen LP, Wessem KJ. Diagnosis and treatment of talar dislocation fractures illustrated by 3 case reports and review of literature. Int J Surg Case Rep 2015;16:106-11. https://doi.org/10.1016/j.ijscr.2015.09.025

12. Schulze W, Richter J, Russe O, Ingelfinger P, Muhr G. Surgical treatment of talus fractures: a retrospective study of 80 cases followed for 1-15 years. Acta Orthop Scand 2002;73(3):344-51. https://doi.org/10.1080/000164702320155374

13. Eberl R, Singer G, Schalamon J, Hausbrandt P, Hoellwarth ME. Fractures of the talus--differences between children and adolescents. J Trauma 2010;68(1):126-30. https://doi.org/10.1097/TA.0b013e3181a74667

14. Smith JT, Curtis TA, Spencer S, Kasser JR, Mahan ST. Complications of talus fractures in children. J Pediatr Orthop 2010;30(8):779-84. https://doi.org/10.1097/BPO.0b013e3181f73e6e

15. Mestdagh H, Duquennoy A, Claisse PR, Sensey JJ, Gougeon F. Long-term prognosis of tarsal dislocations. Arch Orthop Traumatic Surg 1982;99(3):153-9. https://doi.org/10.1007/BF00379202 